\title{
MicroRNA panel in serum reveals novel diagnostic biomarkers for prostate cancer
}

\author{
Shiyu Zhang Equal first author, 1 , Cheng Liu Equal frst author, ${ }^{2}$, Xuan Zou ${ }^{3}$, Xiangnan Geng ${ }^{4}$, Xin Zhou ${ }^{1}$, XingChen Fan ${ }^{1}$, Danxia \\ Zhu ${ }^{5}$, Huo Zhang ${ }^{\text {Corresp., } 6}$, Wei Zhu ${ }^{\text {Corresp. } 1}$ \\ ${ }^{1}$ Department of Oncology, First Affiliated Hospital of Nanjing Medical University, Nanjing, Jiangsu Province, China \\ 2 Department of Gastroenterology, First Affiliated Hospital of Nanjing Medical University, Nanjing, Jiangsu Province, China \\ 3 Fudan University Shanghai Cancer Center, Fudan University Shanghai Cancer Center, Shanghai, Shanghai, China \\ 4 Department of Clinical Engineer, First Affiliated Hospital of Nanjing Medical University, Nanjing, Jiangsu Province, China \\ 5 Department of Oncology, The Third Affiliated Hospital of Soochow University, Changzhou, Jiangsu Province, China \\ 6 Department of Oncology, Northern Jiangsu People's Hospital Affiliated to Yangzhou University, Yangzhou, Jiangsu Province, China \\ Corresponding Authors: Huo Zhang, Wei Zhu \\ Email address: hollyzh1912@126.com, zhuwei@njmu.edu.cn
}

Purpose. MicroRnAs (miRNAs), which could be stably preserved and detected in serum or plasma, could act as biomarkers in cancer diagnosis. Prostate cancer is the second cancer in males for incidence. This study aimed to establish a miRNA panel in peripheral serum which could act as a non-invasive biomarker helping diagnosing PC. Methods. A total of 86 PC patients and 86 normal control serum samples were analyzed through a four-stage experimental process using quantitative real-time polymerase chain reaction. Logistic regression method was used to construct a diagnostic model based on the differentially expressed miRNAs in serum. Receiver operating characteristic curves were constructed to evaluate the diagnostic accuracy. We also compared the 3-miRNA panel with previously reported biomarkers and verified in four public datasets. In addition, the expression characteristics of the identified miRNAs were further explored in tissue and serum exosomes samples. Results. We identified a 3-miRNA signature including up-regulated miR-146a-5p, miR-24-3p and miR-93-5p for PC detection. Areas under the receiver operating characteristic curve of the 3-miRNA panel for the training, testing and external validation phase were $0.819,0.831$ and 0.814 , respectively. The identified signature has a very stable diagnostic performance in the large cohorts of four public datasets. Compared with previously identified miRNA biomarkers, the 3-miRNA signature in this study has superior performance in diagnosing PC. What's more, the expression level of miR-93-5p was also elevated in exosomes from PC samples. However, in PC tissues, none of the three miRNAs showed significantly dysregulated expression. Conclusions. We established a three-miRNA panel (miR-146a-5p, miR-24-3p and miR-93-5p) in peripheral serum which could act as a non-invasive biomarker helping diagnosing PC. 


\section{MicroRNA panel in serum reveals novel diagnostic biomarkers for}

\section{2 prostate cancer}

3 Authors

4 Shiyu Zhang ${ }^{1 \#}$, Cheng Liu ${ }^{2 \#}$, Xuan Zou ${ }^{3}$, Xiangnan Geng ${ }^{4}$, Xin Zhou ${ }^{1}$, Xingchen Fan ${ }^{1}$, Danxia

5 Zhu $^{6}$, Huo Zhang ${ }^{5 *}$, Wei Zhu ${ }^{1 *}$

6 Institution

7 1. Department of Oncology, First Affiliated Hospital of Nanjing Medical University, 300

8 Guangzhou Road, Nanjing 210029, P.R. China;

9 2. Department of Gastroenterology, First Affiliated Hospital of Nanjing Medical University, 300

10 Guangzhou Road, Nanjing 210029, P.R. China;

11 3. Fudan University Shanghai Cancer Center, Xuhui, Shanghai, PR China;

12 4. Department of Clinical Engineer, First Affiliated Hospital of Nanjing Medical

13 University, 300 Guangzhou Road, Nanjing 210029, P.R. China;

14 5. Department of Oncology, Northern Jiangsu People's Hospital Affiliated to Yangzhou

15 University, Yangzhou 225000, China;

16 6. Department of Oncology, The Third Affiliated Hospital of Soochow University, Changzhou

17 213003, P.R. China;

18 \# Contributed equally to this work

19 *Correspondence to Wei Zhu (zhuwei@njmu.edu.cn), Huo Zhang (hollyzh1912@126.com)

Conflict of interest

22 The authors declare that they have no conflict of interest.

Compliance with ethical standards

Ethics approval: All procedures were approved by the Institutional Review Boards of the First Affiliated Hospital of Nanjing Medical University and Jiangsu Cancer Hospital. 
27 Informed consent: Written informed consent was obtained from patients involved in the study.

28

29

30

31

32

33

34

35

36

37

38

39

40

41

42

43

44

45

46

47

48

49

50

51

52

53

Peer] reviewing PDF | (2020:11:55075:2:0:NEW 17 Apr 2021) 
54

\section{Abstract}

Purpose. MicroRNAs (miRNAs) could be stably preserved and detected in serum or plasma and could act as biomarkers in cancer diagnosis. Prostate cancer (PC) is the most frequently diagnosed cancer in men and the second leading cause of male cancer mortality. This study aimed to establish a circulating serum miRNA panel as a noninvasive biomarker for PC.

Methods. The miRNA expression levels of 86 PC patients and 86 normal controls (NCs) in serum were detected by quantitative real-time polymerase chain reaction (qRT-PCR) through a four-stage experimental process. We constructed a logistic regression model based on candidate miRNAs' expression profile to establish an ideal diagnostic miRNA combination. Receiver operating characteristic curves (ROC) were made to evaluate the diagnostic accuracy. We also compared the diagnostic value of the 3-miRNA panel with previously reported biomarkers and verified them in four public datasets. Also, to explore the identified miRNAs' potential existence, we detected miRNA expressions in tissue and the matched adjacent normal tissue samples and exosome samples.

Results. We identified a miRNA signature of three up-regulated miRNAs (miR-146a-5p, miR24-3p, and miR-93-5p). The three-miRNA panel's areas under the receiver operating characteristic curve (AUC) were $0.819,0.831$, and 0.814 for the three experimental phases (the training, testing, and validation stages). The diagnostic performance of the identified 3-miRNA signature shows stability in the large cohorts of the four public datasets and superior to previously identified miRNA biomarkers. What is more, the expression level of miR-93-5p was also elevated in exosomes in PC samples. However, in PC tissue samples, none of the three miRNAs showed significantly dysregulated expression.

Conclusions. We established a three-miRNA panel (miR-146a-5p, miR-24-3p, and miR-93-5p) which could act as a noninvasive serum biomarker to diagnose PC.

Keyword. miRNAs; prostate cancer; diagnostic biomarker; qRT-PCR. 


\section{Introduction}

Prostate cancer (PC) is the most frequently diagnosed cancer in men and the second leading cause of male cancer mortality [1]. The vast majority of PC, approximately $95 \%$, presents clinically localized to the prostate without definite metastasis evidence, the 5-year survival of which was almost 100\% [2]. The initial clinical assessment of the primary tumor has been based on digital rectal examination findings, the serum prostate-specific antigen (PSA) level, and histological confirmation of PC [3]. However, these methods have some limitations, such as a low predictive value and the adverse consequences of overdiagnosis and overtreatment [4]. Thus, the exploration of novel noninvasive biomarkers with high sensitivity and specificity for PC diagnosis is urgently needed.

MicroRNAs (miRNAs) are small non-coding RNAs (19-22 nucleotides) that could negatively regulate gene expression at the post-transcriptional level by binding the 3'-UTR of target mRNAs, leading to mRNA degradation and translation repression [5]. Several miRNAs have been reported to be stably detected in serum or plasma, suggesting their potential values as new biomarkers for early diagnosis of various kinds of cancers [6-12]. Meanwhile, researchers found that some miRNAs could discriminate PC patients from normal controls (NCs), thus working as peripheral circulation biomarkers for PC screening [13-16]. However, the results of researches exploring miRNAs in PC detection were found inconsistent to some extent. There are many reasons for the inconsistency, such as variation of patient characteristics, miRNA analysis techniques, methods of extracting miRNAs, etc. We want to construct a miRNA expression profile of pan-cancer of ourselves.

In the present study, we focused on PC patients and conducted a four-stage study with qRT-PCR to find a potential miRNA profile for detecting PC. We then compared the identified miRNA panel with some other signatures that were previously reported to verify its diagnostic performance. We also did some bioinformatics analysis to confirm our result [17-20]. What is more, the expression of selected miRNAs in tissues and serum exosomes were evaluated to explore their sources and potential forms in circulation. This paper displays preliminary results 
and will be further verified among larger samples with the more statistical process before clinical application. We hope that our findings may contribute to the construction of promising biomarkers for PC detection.

\section{Materials and methods}

\subsection{Study design, patients, and samples}

Subjects enrolled in the study were recruited from the Third Affiliated Hospital of Soochow University, Jiangsu Cancer Hospital, and the First Affiliated Hospital of Nanjing Medical University between 2016 and 2017. The Institutional Review Boards approved all procedures of the First Affiliated Hospital of Nanjing Medical University (Ethical Application Ref: 2016SRFA-149). We obtained the information of TNM stage, differentiation degree, and clinical characteristics from patients' records (according to the seventh edition American Joint Committee on Cancer (AJCC)). All the PC patients were pathologically confirmed. We drew blood samples when patients did not receive any therapeutic procedures like surgery, endocrine therapy, or chemotherapy. NCs enrolled in our study were healthy volunteers who took the routine physical examination at the First Affiliated Hospital of Nanjing Medical University. Each participant provided the written informed consent.

A total of 86 PC patients and 86 normal controls were enrolled in our study. As shown in Fig.1, we designed a four-stage experimental process. Several factors were taken into account in sample selection and distribution: (i) the purpose of each separate stage; (ii) the sequence of sample collection in practical operation; (iii) the balance of non-experimental factors among four different sets; (iv) the basic principle of experiment design — control, randomization, replication, and balance. Also, factors such as gender, age, TNM stage, and pathological type might have confounding effects on circulating miRNA expression in PC. Therefore, these potential interference factors were as evenly distributed across four sets as possible to avoid selection bias. In the initial screening phase, we randomly chose twenty peripheral serum samples from PC patients, ten from NCs, and then synthesized two PC sample pools and 1 NC sample pool for miRNA profiling (we pooled every ten samples as one pool sample). 
135 Then, to verify the reproducibility and reliability of the results acquired from the screening

136 phase, serum samples from 28 PC patients and 28 NCs were used to confirm the dysregulated

137 miRNAs in the training stage. The miRNAs identified by the training stage were validated in the

138 following testing stage in $32 \mathrm{PC}$ patients and $32 \mathrm{NCs}$. After the three steps above, we set an

139 external validation phase to further assess the three-miRNA signature's diagnostic value with 26

140 PC patients and 26 NCs. As shown in table 1, no significant difference in the distribution of age

141 between PC and NC samples was found in any stage. As previously described in our team's 142 another study [21], the identified miRNAs were further validated in 28 pairs of formalin-fixed 143 paraffin-embedded (FFPE) PC tissues and matched adjacent normal tissue specimens from the 144 same surgery patients. Additionally, we analyzed miRNAs' expression in exosomes in 24 PC 145 patients and $24 \mathrm{NCs}$ to investigate the miRNAs' potential form in the peripheral circulation.

146 We collected $5 \mathrm{ml}$ of venous blood in a vacuum blood collection tube and then put them in a 147 refrigerator at $4^{\circ} \mathrm{C}$. These blood collections were processed within 12 hours using a two-step 148 centrifugation method $\left(1,500 \mathrm{rpm}^{*} 10 \mathrm{~min}\right.$, then $\left.12,000 \mathrm{rpm}^{*} 2 \mathrm{~min}\right)$ at $4^{\circ} \mathrm{C}$. Next, we carefully 149 aspirated and separated the supernatant to obtain the serum, aliquoted it into clean, enzyme-free 150 EP tubes to prevent repeated freezing and thawing. These serum samples were then stored in a $15180^{\circ} \mathrm{C}$ refrigerator for further analysis.

$152 \quad 2.2$ Exosomes isolation

153 According to the manufacturer's protocol, exosomes were isolated from serum using 154 ExoQuick $^{\mathrm{TM}}$ (System Biosciences, Mountain View, Calif). Briefly, 200 $\mu$ l serum was mixed with $155100 \mu \mathrm{l}$ ExoQuick exosome precipitation solution. Then we precipitated exosome pellets by 156 centrifugation at $13,000 \mathrm{rpm}$ for $2 \mathrm{~min}$. The obtained exosome samples were next lysed into $200 \mu 1$ 157 RNAse-free water for further analysis.

\subsection{RNA extraction}

As previously described [22], total RNA was isolated from $200 \mu 1$ serum or exosome samples using mirVana Paris Kit (Ambion, Austin, TX, USA) under the manufacturer's protocol instruction. We added $5 \mu$ l of synthetic C. elegans miR-39 (5nM/L, RiboBio, Guangzhou, China) 
162 to each sample after denaturing the solution (Ambion, Austin, TX, USA) for sample-to-sample normalization. We extracted total RNA from FFPE specimens via the High Pure FFPE RNA Micro Kit (Ambion Austin, TX, USA). Then, we dissolved RNA in 100 $\mu$ l RNAse-free water and stored these RNA samples at $-80^{\circ} \mathrm{C}$ until use. Nanodrop 2000 Thermo scientific spectrophotometer (NanoDrop Technologies, Wilmington, DE, USA) was used to evaluate the total RNA's concentration and purity.

\subsection{Quantitative reverse transcription polymerase chain reaction (qRT-PCR)}

The experimental process was mentioned in the previous article [22]. MiRNA was reverse transcribed to cDNA using the miRCURY Locked Nucleic Acid (LNATM) Universal Reverse Transcription (RT) microRNA PCR, Polyadenylation, and cDNA synthesis kit (Exiqon miRNA qPCR panel, Vedbaek, Denmark). We then scanned the microarrays on a 7900HT real-time PCR system (Applied Biosystems, Foster City, CA, USA) with Exiqon miRCURY-Ready-to-UsePCR-Human-panel-I + II-V1.M (Exiqon miRNA qPCR panel, Vedbaek, Denmark). The amplification of miRNA was performed via specific primers of reverse transcription (RT) and polymerase chain reaction (PCR) (RiboBio, Guangzhou, China). The RT reaction was performed at $42^{\circ} \mathrm{C}$ for $60 \mathrm{~min}$ followed by $70^{\circ} \mathrm{C}$ for $10 \mathrm{~min}$; the PCR reaction was performed at $95^{\circ} \mathrm{C}$ for 20 $\mathrm{sec}$, followed by 40 cycles of $95^{\circ} \mathrm{C}$ for $10 \mathrm{sec}, 60^{\circ} \mathrm{C}$ for $20 \mathrm{sec}$ and then $70^{\circ} \mathrm{C}$ for $10 \mathrm{sec}$ on a 7900HT real-time PCR system. qRT-PCR was performed to amplify and detect the miRNAs with SYBR Green Dye (SYBR ® Premix Ex TaqTM II, TaKaRa, Dalian, China). The expression of miRNAs in serum samples and exosomes were calculated using the comparative $2^{-\Delta \Delta \mathrm{Ct}}$ method normalized to exogenous reference miRNA (cel-miR-103a-3p), $\Delta \mathrm{Ct}=\mathrm{CtmiRNA}-\mathrm{Ctcel}-$ miR-103a-3p. Tissue miRNAs were determined by the $2^{-\Delta \Delta C t}$ method relative to cel-miR-39 and RNU6B (U6) (Livak and Schmittgen, 2001).

\subsection{Statistical analysis}

Mann-Whitney test was used to analyze the differential expression of miRNAs between PC patients and NCs. Moreover, the association between miRNAs and the clinical characteristics was estimated using one-way ANOVA or $\chi^{2}$ test. Sample size calculation has been done using 
software PASS. A sample of 12 from the positive group and 12 from the negative group achieves $91 \%$ power to detect a difference of 0.3 between the area under the ROC curve (AUC) under the null hypothesis of 0.5 and an AUC under the alternative hypothesis of 0.8 using a two-sided ztest at a significance level of 0.15 . The receiver operating characteristic (ROC) curves and the area under the ROC curve (AUC) calculation, along with Cox's regression models, were applied to estimate the diagnostic and prognostic value of identified miRNAs. A logistic regression model for PC prediction was used to the data of the training and validation stages. SPSS (version 19.0, IBM, Armonk, New York, USA) and GraphPad Prism 7 (GraphPad Software, USA) were used to performed statistical analyses. Differences were considered significant when P-value $<0.05$.

\subsection{Bioinformatics analysis}

Pathway enrichment analyses of miRNA gene targets and differentially expressed genes were performed using DIANA-mirPath v.3.0 (http://www.microrna.gr,miRPathv3) [23]. We conducted Gene Ontology (GO) [17] analysis and Kyoto Encyclopedia of Genes and Genomes (KEGG) [18] analysis to predict possible biological processes and potential signal pathways. To further confirm our results, we downloaded prostate adenocarcinoma data from The Cancer Genome Atlas (TCGA) and performed a similar analysis.

\section{Results}

\subsection{Discovery of candidate miRNAs from the screening phase}

To filter candidate miRNAs in the screening phase, we screen the expression levels of 168 miRNAs in the 2 PC sample pools and the NC sample pool using Exiqon miRCURY-Ready-toUse-PCR-Human-panel-I + II-V1.M on the qRT-PCR platform. Screening criteria for candidate miRNAs were as previously described[22]. Only the miRNAs with cycle threshold (Ct) values $<$ 37 and 5 lower than negative control in the panel were eligible for further analysis. A miRNA was considered as a candidate miRNA if it showed more than 1.5-fold or less than 0.67-fold altered expression in both two pooled PC samples compared to the NC pool sample. As shown in table 2, a total of 37 miRNAs was found to exhibit dysregulated expression in PC and chosen for 
216 the further training stage.

\section{$217 \quad 3.2$ Confirmation of identified miRNAs by qRT-PCR}

218 The expression of the 37 candidate miRNAs selected via the screening phase was further 219 analyzed in 28 PC patients and 28 NCs using qRT-PCR in the training phase. 4 of the 37

220 miRNAs were further confirmed. During the testing stage, all four miRNAs were subsequently

221 analyzed in 32 PC patient samples and 32 NCs. 3 of the 4 show consistently up-regulated

222 expression and met our standards (Table 3). We then assessed the three miRNAs (miR-146a-5p, miR-24-3p, and miR-93-5p) in 26 PC patient samples and $26 \mathrm{NCs}$ in an external validation stage and found that the result was the same as above. What's more, all the three miRNAs had significantly higher expression levels in peripheral serum of PC patients as compared with NCs when the results of the three stages were combined (Fig.2).

\subsection{Diagnostic value of the candidate miRNAs}

To evaluate the three miRNAs' performance in PC diagnosis, we conducted ROC curves on each of the three miRNAs. Taken three phases into consideration (training phase, testing phase and external validation phase), the AUC for serum miR-146a-5p, miR-24-3p and miR-93-5p was 0.767 (95\% confidence interval (CI): 0.696-0.839), 0.767 (95\% CI: 0.694-0.840) and 0.781 (95\% CI: $0.710-0.852$ ), respectively (Fig.S1). Therefore, each of these three miRNAs had the ability to distinguish PC patients from healthy controls. In addition, when these three miRNAs were treated as a whole, we found that this 3-miRNA signature had a better diagnostic performance. As shown in Fig.3A, combining the three stages, the AUC of this miRNA panel was 0.819 (95\% CI: $0.754-0.885$; sensitivity $75.6 \%$, specificity $85.7 \%$ ). Meanwhile, the diagnostic value of the 3miRNA panel was assessed in a combination of the former two cohorts (the training and testing stage). The AUC was 0.831 (95\% CI: 0.757-0.905; sensitivity $=73.3 \%$, specificity $=80.0 \%$; Fig.3B). In the external validation stage, similar to the previous training and testing phases, the three identified miRNAs showed a consistent tendency of upregulation, and the panel still performed quite well in discriminating PC patients from NCs with the AUC being AUC of 0.814 (95\% CI: $0.687-0.942$; sensitivity $=76.9 \%$ and specificity $=83.3 \%$; Fig. $3 \mathrm{C})$. The correlation 
243 between the serum PSA values, TNM stages, and the three serum miRNAs levels was also

244 analyzed, respectively. However, no significant results were observed. Therefore, data were not 245 presented.

\section{3.4 External validation in public databases}

247 We further verified the diagnostic value of the 3-miRNA signature in four public datasets: The 248 Cancer Genome Atlas Prostate Adenocarcinoma (TCGA-PRAD) dataset

249 (http://cancergenome.nih.gov/), GSE113740 dataset, GSE113486 dataset, and GSE112264

250 dataset. The first TCGA-PRAD dataset represents data from tissues, while the other three 251 datasets contain data of serum samples. In the TCGA-PRAD dataset, the 3-miRNA marker 252 performed well in distinguishing between PC tumor tissues $(n=498)$ and normal tissues $(n=51)$. 253 As shown in supplementary table S1, the corresponding AUC of the 3-miRNA signature was

\subsection{Prognostic value of the three miRNAs for PC}


270 Using data from the TCGA-PRAD dataset, we constructed Cox regression models to estimate the 271 correlation of several influence factors and overall survival (OS) and the three serum miRNAs'

272 prognostic value for PC. However, none of the three miRNAs were significantly associated with 273 prognosis with $\mathrm{P}>0.05$ (table S2).

274

275

\subsection{Expression of the miRNAs in tissue samples}

We next detected the expression levels of the three miRNAs in 28 pairs of tissue samples by qRT-PCR. As shown in Fig.S3, the results were not statistically significant in this stage. However, when we analyzed the three miRNAs in the un-matched tissue samples with larger sample size through The Cancer Genome Atlas (TCGA), as shown in Fig.5, miR-146a-5p and miR-93-5p were found to be consistent with those in serum. In contrast, miR-24-3p was found down-regulated compared with NCs, which was opposite to the expression level of miR-24-3p in serum.

\subsection{Expression of the identified miRNAs in serum exosomes}

Additionally, the expression levels of miRNAs in exosomes were assessed in 24 PC patient samples and 24 NCs by qRT-PCR to explore the potential existing form of the identified miRNAs in PC patients' serum. Compared to NCs, all the three miRNAs were up-regulated, but only miR-93-5p was statistically significant in serum exosomes (Fig.6).

\subsection{Bioinformatics analysis of the identified miRNAs}

We assessed these experimentally identified miRNAs with DIANA-miRPathv3.0, a web-server aiming for pathway analysis. By performing the Kyoto Encyclopedia of Genes and Genomes (KEGG) and Gene Ontology (GO) analyses, we analyzed the validated miRNAs' potential roles that might take part. As shown in Fig.S4A, KEGG analysis showed that these identified miRNAs might participate in some pathways such as AMPK signaling pathway, pathway in cancer, MAPK signaling pathway, TGF-beta signaling pathway, p53 signaling pathway, and so on. As demonstrated in Fig.S4B, GO analysis showed that in RNA metabolic activity, cell cycle, cell proliferation, cell death, and many other biological processes, the three miRNAs should not be ignored. 
297

298

299

300

301

302

303

304

305

306

307

\section{Discussion}

In the present study, we carefully designed a procedure to identify serum miRNAs with potential for PC detection. In the screening stage, we analyzed differential expression profiles of miRNAs in $2 \mathrm{PC}$ and $1 \mathrm{NC}$ serum pools using Exiqon miRNA qPCR Panels, which showed more sensitivity and linear compared to the TaqMan platform [30]. Then, we conducted three phases for validation to control the false-positive rate (the training stage, the testing stage, and the external validation stage) based on qRT-PCR subsequently. Ultimately, based on a cohort of 172 serum samples, including 86 PC patients and $86 \mathrm{NCs}$, three up-regulated serum miRNAs (miR146a-5p, miR-24-3p, and miR-93-5p) were identified, which simultaneously proved to have a high accuracy in PC diagnosis. Compared with a single miRNA, the molecular signature of the 3-miRNA combination could provide more reliable information about the disease status, and this expression profile might become a diagnostic molecular signature for prostate cancer in the future.

To further confirm the diagnostic value of the 3-miRNA label, we checked previously published articles that similarly identify serum miRNAs as diagnostic biomarkers for PC. It's important to note that these biomarkers should distinguish PC patients from healthy people rather than differentiate tumor tissue from normal tissue. Moreover, only serum miRNAs were included for the analysis to ensure comparability. Several studies have identified circulating miRNAs as promising biomarkers in the diagnosis of PC [14-16, 31-33]. However, we could see that conclusions showed inconsistent results from variations of patient characteristics, techniques for miRNA analysis, and methods of extracting miRNAs [33]. While incorporating this 3-miRNA signature with some of the previously identified markers may yield a higher sensitivity or specificity for PC diagnosis, most of these miRNAs were not detected by qRT-PCR in our study because they failed to meet the criteria in the screening stage.

By consulting the previous literature, we learned the relevant information about these three miRNAs. MiR-146a-5p played a vital role in the process of androgen-independent prostate cancer cell apoptosis by targeting the ROCK/Caspase 3 pathway [34]. Sun Q et al. [35] 
324

325

326

327

328

329

330

331

332

333

334

335

336

337

338

339

340

341

342

343

344

345

346

347

348

349

350

suggested that miR-146a-5p acted as a cancer suppressor through down-regulation of Rac1. Meanwhile, Daniel R et al. [31] identified another miRNA panel as a diagnostic biomarker, including miR-146a-5p, which confirmed our current research to a certain extent. Circulating miR-146a-5p was reported to show dysregulation in various types of tumors, such as oral squamous cell carcinoma [36], breast cancer [37], non-small cell lung cancer [38], bladder cancer, and many other types of tumor [39]. Fredsøe, J et al. [40] suggested that the up-regulated expression of miR-24-3p in urine made it possible to be a biomarker for PC detection. Cochetti, $\mathrm{G}$ et al. [32] also analyzed circulating miR-24-3p between PC patients and people with benign prostatic hyperplasia but came to a negative conclusion. There were too many other studies supporting that miR-24-3p functioned in various types of tumor except for PC. Kang, $\mathrm{H}$ et al. [41] suggested that miR-24-3p could function as a tumor suppressor by targeting p130Cas in metastatic cancer. Gao, Y et al. [42] revealed that over-expression of miR-24-3p could suppress colorectal cancer cell proliferation, cell migration, and invasion. However, reports also showed that miR-24-3p might promote cell migration and proliferation in lung cancer [43] and bladder cancer [44]. Khodadadi-Jamayran, A et al. [45] found that patients with breast cancer, compared to those with low miR-24-3p levels, patients with the primarily high expression level miR-24-3p seemed to have a lower survival rate. As for head and neck squamous cell carcinoma, Sun, X et al. [46] believe that miR-24-3p could promote cell proliferation and regulate chemosensitivity. What's more, Meng, F. L et al. [47] suggested that miR-24-3p could be viewed as an independent predictor of poor overall survival and disease-free survival in patients with HBV-related hepatocellular carcinoma. Yang, Y. [48] suggested that miR-93-5p might be an oncogene in PC. More studies are still needed to explore how miR-93-5p functions during the life circle of prostate cancer. Researches were demonstrating that miR-93-5p could promote gastric cancer metastasis through inactivating the Hippo signaling pathway [49] and, on the other side, activating the STAT3 signaling pathway [50]. Xiang, Y et al. [51] suggested that via targeting MKL-1 and STAT3 signaling pathway, miR-93-5p could inhibit the epithelial-mesenchymal transition. Li, J et al. [52] suggested that as to high-risk HPV-positive cervical cancer, 
351

352

353

354

355

356

357

358

359

360

361

362

363

364

365

366

367

368

369

370

371

372

373

374

375

376

377

suppression of miR-93-5p might inhibit cancer cell progression by targeting BTG3. MiR-93-5p may also play an essential role via down-regulating PPARGC1A in hepatocellular carcinoma cells [53].

It was reported that circulating miRNAs might be released from tumor tissues [33]. Hence, we explored the three serum miRNAs' expression levels in PC tissues and corresponding adjacent normal tissues. Unfortunately, the result didn't achieve statistical significance. However, data of a larger sample size of unpaired tumor tissue and normal tissues from TCGA showed that miR146a-5p and miR-93-5p were consistent with the miRNAs' expression in serum of our study, while miR-24-3p was the opposite. These suggested that the three miRNAs were closely related to $\mathrm{PC}$.

Moreover, studies showed that circulating miRNAs could be embedded in exosomes or bound with Ago1-4 proteins independent from small membrane vesicles $[54,55]$. In this study, we also identified serum miRNAs in exosomes to find the potential form of the three selected miRNAs. All the three miRNAs in serum exosomes showed high expression levels, but only miR-93-5p was presented with statistical significance in PC compared to NCs. A relatively small sample size might be partly responsible for the results.

There are still some limitations that we should consider. Firstly, more work needs to be done in the future to explore the value of the three identified miRNAs in assessing tumor dynamics. Secondly, how these miRNAs exactly play their roles in PC is not clear, and further investigations are needed.

In conclusion, we identified a three-miRNA panel for PC detection. This study displays preliminary results and will be further verified among larger samples with more statistical processes clinical application in the future. We believe that this work will serve as the basis of circulating miRNAs in clinical for the diagnosis of PC in the future, at least for the Asian population to a certain extent.

\section{Conclusion}

To summarize, we have identified a three-miRNA panel (miR-146a-5p, miR-24-3p, and miR-93- 
378

379

380

381

382

383

384

385

386

387

388

389

390

391

392

393

394

395

396

397

398

399

400

401

402

403

404

405

406

407

408

409

410

411

412

413

414

415

$5 p)$ for the detection of PC in serum. This work can lay the foundation for clinical diagnosis of PC in the future. Still, larger cohorts of participants are needed to focus on the three miRNAs' mechanisms in PC.

\section{Acknowledgments}

I would like to show my deepest gratitude to all volunteers involved in this study. Their understanding and dedication enable medicine to explore and advance continuously.

\section{REFERENCES}

1. Bray, F., J. Ferlay, I. Soerjomataram, R.L. Siegel, L.A. Torre, and A. Jemal, Global cancer statistics 2018: GLOBOCAN estimates of incidence and mortality worldwide for 36 cancers in 185 countries. CA Cancer J Clin, 2018. 68(6): p. 394-424.

2. Siegel, R.L., K.D. Miller, and A. Jemal, Cancer Statistics, 2017. CA Cancer J Clin, 2017. 67(1): p. 7-30.

3. Buyyounouski, M.K., P.L. Choyke, J.K. McKenney, O. Sartor, H.M. Sandler, M.B. Amin, M.W. Kattan, and D.W. Lin, Prostate cancer - major changes in the American Joint Committee on Cancer eighth edition cancer staging manual. CA Cancer J Clin, 2017. 67(3): p. 245-253.

4. Grossman, D.C., S.J. Curry, D.K. Owens, K. Bibbins-Domingo, A.B. Caughey, K.W. Davidson, C.A. Doubeni, M. Ebell, J.W. Epling, A.R. Kemper, A.H. Krist, M. Kubik, C.S. Landefeld, C.M. Mangione, M. Silverstein, M.A. Simon, A.L. Siu, and C.W. Tseng, Screening for Prostate Cancer: US Preventive Services Task Force Recommendation Statement. Jama, 2018. 319(18): p. 1901-1913.

5. $\quad$ Bartel, D.P., Metazoan MicroRNAs. Cell, 2018. 173(1): p. 20-51.

6. Carter, J.V., N.J. Galbraith, D. Yang, J.F. Burton, S.P. Walker, and S. Galandiuk, Bloodbased microRNAs as biomarkers for the diagnosis of colorectal cancer: a systematic review and meta-analysis. Br. J. Cancer, 2017. 116(6): p. 762-774.

7. Huang, Z., D. Zhu, L. Wu, M. He, X. Zhou, L. Zhang, H. Zhang, W. Wang, J. Zhu, W. Cheng, Y. Chen, Y. Fan, L. Qi, Y. Yin, W. Zhu, Y. Shu, and P. Liu, Six Serum-Based miRNAs as Potential Diagnostic Biomarkers for Gastric Cancer. Cancer Epidemiol. Biomarkers Prev., 2017. 26(2): p. 188-196.

8. Zhang, H., M. Zhu, X. Shan, X. Zhou, T. Wang, J. Zhang, J. Tao, W. Cheng, G. Chen, J. $\mathrm{Li}, \mathrm{P}$. Liu, Q. Wang, and W. Zhu, A panel of seven-miRNA signature in plasma as potential biomarker for colorectal cancer diagnosis. Gene, 2019. 687: p. 246-254.

9. Zhou, X., W. Wen, X. Shan, W. Zhu, J. Xu, R. Guo, W. Cheng, F. Wang, L.W. Qi, Y. Chen, Z. Huang, T. Wang, D. Zhu, P. Liu, and Y. Shu, A six-microRNA panel in plasma was identified as a potential biomarker for lung adenocarcinoma diagnosis. Oncotarget, 2017. 8(4): p. 6513-6525. 
417

418

419

420

421

422

423

424

425

426

427

428

429

430

431

432

433

434

435

436

437

438

439

440

441

442

443

444

445

446

447

448

449

450

451

452

453

454

455

456
416 10. Zhou, X., W. Zhu, H. Li, W. Wen, W. Cheng, F. Wang, Y. Wu, L. Qi, Y. Fan, Y. Chen, Y. Ding, J. Xu, J. Qian, Z. Huang, T. Wang, D. Zhu, Y. Shu, and P. Liu, Diagnostic value of a plasma microRNA signature in gastric cancer: a microRNA expression analysis. Sci Rep, 2015. 5: p. 11251.

11. Zhu, M., Z. Huang, D. Zhu, X. Zhou, X. Shan, L.W. Qi, L. Wu, W. Cheng, J. Zhu, L. Zhang, H. Zhang, Y. Chen, W. Zhu, T. Wang, and P. Liu, A panel of microRNA signature in serum for colorectal cancer diagnosis. Oncotarget, 2017. 8(10): p. 17081-17091.

12. Wang, J., H. Zhang, X. Zhou, T. Wang, J. Zhang, W. Zhu, H. Zhu, and W. Cheng, Five serum-based miRNAs were identified as potential diagnostic biomarkers in gastric cardia adenocarcinoma. Cancer Biomark, 2018. 23(2): p. 193-203.

13. Shukla, K.K., S. Misra, P. Pareek, V. Mishra, B. Singhal, and P. Sharma, Recent scenario of microRNA as diagnostic and prognostic biomarkers of prostate cancer. Urol. Oncol., 2017. 35(3): p. 92-101.

14. Kanwal, R., A.R. Plaga, X. Liu, G.C. Shukla, and S. Gupta, MicroRNAs in prostate cancer: Functional role as biomarkers. Cancer Lett., 2017. 407: p. 9-20.

15. Moustafa, A.A., M. Ziada, A. Elshaikh, A. Datta, H. Kim, K. Moroz, S. Srivastav, R. Thomas, J.L. Silberstein, K. Moparty, F.E. Salem, O.H. El-Habit, and A.B. Abdel-Mageed, Identification of microRNA signature and potential pathway targets in prostate cancer. Exp. Biol. Med. (Maywood), 2017. 242(5): p. 536-546.

16. Kumar, B. and S.E. Lupold, MicroRNA expression and function in prostate cancer: $a$ review of current knowledge and opportunities for discovery. Asian J. Androl. 18(4): p. 559-67.

17. The Gene Ontology Resource: 20 years and still GOing strong. Nucleic acids research, 2019. 47(D1): p. D330-D338.

18. Kanehisa, M. and S. Goto, KEGG: kyoto encyclopedia of genes and genomes. Nucleic acids research, 2000. 28(1): p. 27-30.

19. Le, N.Q.K., Fertility-GRU: Identifying Fertility-Related Proteins by Incorporating DeepGated Recurrent Units and Original Position-Specific Scoring Matrix Profiles. Journal of proteome research, 2019. 18(9): p. 3503-3511.

20. Le, N.Q.K., E.K.Y. Yapp, N. Nagasundaram, and H.-Y. Yeh, Classifying Promoters by Interpreting the Hidden Information of DNA Sequences via Deep Learning and Combination of Continuous FastText $\mathrm{N}$-Grams. Frontiers in bioengineering and biotechnology, 2019. 7: p. 305.

21. Shan, X., H. Zhang, L. Zhang, X. Zhou, T. Wang, J. Zhang, Y. Shu, W. Zhu, W. Wen, and P. Liu, Identification of four plasma microRNAs as potential biomarkers in the diagnosis of male lung squamous cell carcinoma patients in China. Cancer Med, 2018. 7(6): p. 23702381.

22. Zhou, X., W. Wen, J. Zhu, Z. Huang, L. Zhang, H. Zhang, L.W. Qi, X. Shan, T. Wang, W. Cheng, D. Zhu, Y. Yin, Y. Chen, W. Zhu, Y. Shu, and P. Liu, A six-microRNA signature in plasma was identified as a potential biomarker in diagnosis of esophageal squamous cell carcinoma. Oncotarget, 2017. 8(21): p. 34468-34480. 
457 23. Vlachos, I.S., K. Zagganas, M.D. Paraskevopoulou, G. Georgakilas, D. Karagkouni, T. Vergoulis, T. Dalamagas, and A.G. Hatzigeorgiou, DIANA-miRPath v3.0: deciphering microRNA function with experimental support. Nucleic acids research, 2015. 43(W1): p. W460-W466.

24. Porzycki, P., E. Ciszkowicz, M. Semik, and M. Tyrka, Combination of three miRNA (miR141, miR-21, and miR-375) as potential diagnostic tool for prostate cancer recognition. Int Urol Nephrol, 2018. 50(9): p. 1619-1626.

25. Moltzahn, F., A.B. Olshen, L. Baehner, A. Peek, L. Fong, H. Stöppler, J. Simko, J.F. Hilton, P. Carroll, and R. Blelloch, Microfluidic-Based Multiplex qRT-PCR Identifies Diagnostic and Prognostic microRNA Signatures in the Sera of Prostate Cancer Patients. Cancer Research, 2011. 71(2): p. 550-560.

26. Chen, Z.H., G.L. Zhang, H.R. Li, J.D. Luo, Z.X. Li, G.M. Chen, and J. Yang, A panel of five circulating microRNAs as potential biomarkers for prostate cancer. Prostate, 2012. 72(13): p. 1443-52.

27. Bryant, R.J., T. Pawlowski, J.W. Catto, G. Marsden, R.L. Vessella, B. Rhees, C. Kuslich, T. Visakorpi, and F.C. Hamdy, Changes in circulating microRNA levels associated with prostate cancer. Br J Cancer, 2012. 106(4): p. 768-74.

28. Yaman Agaoglu, F., M. Kovancilar, Y. Dizdar, E. Darendeliler, S. Holdenrieder, N. Dalay, and U. Gezer, Investigation of miR-21, miR-141, and miR-221 in blood circulation of patients with prostate cancer. Tumour biology : the journal of the International Society for Oncodevelopmental Biology and Medicine, 2011. 32(3): p. 583-588.

29. Paunescu, I.A., R. Bardan, A. Marcu, D. Nitusca, A. Dema, S. Negru, O. Balacescu, L. Balacescu, A. Cumpanas, I.O. Sirbu, B. Petrut, E. Seclaman, and C. Marian, Biomarker Potential of Plasma MicroRNA-150-5p in Prostate Cancer. Medicina, 2019. 55(9): p. 564.

30. Steffen G Jensen, P.L., Mads H Rasmussen, Marie S Ostenfeld, Lars Dyrskjøt, Torben F Ørntoft and Claus L Andersen, Evaluation of two commercial global miRNAexpression profiling platforms for detection ofless abundant miRNAs. BMC Genomics, 2011. 12: p. 435.

31. Daniel, R., Q. Wu, V. Williams, G. Clark, G. Guruli, and Z. Zehner, A Panel of MicroRNAs as Diagnostic Biomarkers for the Identification of Prostate Cancer. Int J Mol Sci, 2017. 18(6).

32. Cochetti, G., G. Poli, G. Guelfi, A. Boni, M.G. Egidi, and E. Mearini, Different levels of serum microRNAs in prostate cancer and benign prostatic hyperplasia: evaluation of potential diagnostic and prognostic role. Onco Targets Ther, 2016. 9: p. 7545-7553.

33. Endzeliņš, E., V. Melne, Z. Kalniņa, V. Lietuvietis, U. Riekstiṇa, A. Llorente, and A. Linē, Diagnostic, prognostic and predictive value of cell-free miRNAs in prostate cancer: $a$ systematic review. Mol. Cancer, 2016. 15(1): p. 41.

34. Xu, B., Y. Huang, X. Niu, T. Tao, L. Jiang, N. Tong, S. Chen, N. Liu, W. Zhu, and M. Chen, Hsa-miR-146a-5p modulates androgen-independent prostate cancer cells apoptosis by targeting ROCK1. Prostate, 2015. 75(16): p. 1896-903.

35. Sun, H., S. Liu, Y. Zhang, and M.R. Whittle, Comparison of southern Chinese Han and 
Brazilian Caucasian mutation rates at autosomal short tandem repeat loci used in human forensic genetics. International journal of legal medicine, 2014. 128(1): p. 1-9.

36. Min, S.K., S.Y. Jung, H.K. Kang, S.A. Park, J.H. Lee, M.J. Kim, and B.M. Min, Functional diversity of miR-146a-5p and TRAF6 in normal and oral cancer cells. Int. J. Oncol., 2017. 51(5): p. 1541-1552.

37. Si, C., Q. Yu, and Y. Yao, Effect of miR-146a-5p on proliferation and metastasis of triplenegative breast cancer via regulation of SOX5. Exp Ther Med, 2018. 15(5): p. 4515-4521.

38. Yuwen, D.L., B.B. Sheng, J. Liu, W. Wenyu, and Y.Q. Shu, MiR-146a-5p level in serum exosomes predicts therapeutic effect of cisplatin in non-small cell lung cancer. Eur Rev Med Pharmacol Sci, 2017. 21(11): p. 2650-2658.

39. Iacona, J.R. and C.S. Lutz, miR-146a-5p: Expression, regulation, and functions in cancer. Wiley Interdiscip Rev RNA, 2019. 10(4): p. e1533.

40. Fredsøe, J., A.K.I. Rasmussen, E.B. Laursen, Y. Cai, K.A. Howard, B.G. Pedersen, M. Borre, P. Mouritzen, T. Ørntoft, and K.D. Sørensen, Independent Validation of a Diagnostic Noninvasive 3-MicroRNA Ratio Model O for Prostate Cancer in Cell-Free Urine. Clin. Chem., 2019. 65(4): p. 540-548.

41. Kang, H., J.G. Rho, C. Kim, H. Tak, H. Lee, E. Ji, S. Ahn, A.R. Shin, H.I. Cho, Y.H. Huh, W.K. Song, W. Kim, and E.K. Lee, The miR-24-3p/p130Cas: a novel axis regulating the migration and invasion of cancer cells. Sci Rep, 2017. 7: p. 44847.

42. Gao, Y., Y. Liu, L. Du, J. Li, A. Qu, X. Zhang, L. Wang, and C. Wang, Down-regulation of miR-24-3p in colorectal cancer is associated with malignant behavior. Med. Oncol., 2015. 32(1): p. 362.

43. Yan, L., J. Ma, Y. Zhu, J. Zan, Z. Wang, L. Ling, Q. Li, J. Lv, S. Qi, Y. Cao, Y. Liu, L. Cao, Y. Zhang, Z. Qi, and L. Nie, miR-24-3p promotes cell migration and proliferation in lung cancer by targeting SOX7. J. Cell. Biochem., 2018. 119(5): p. 3989-3998.

44. Yu, G., Z. Jia, and Z. Dou, miR-24-3p regulates bladder cancer cell proliferation, migration, invasion and autophagy by targeting DEDD. Oncol. Rep., 2017. 37(2): p. 11231131.

45. Khodadadi-Jamayran, A., B. Akgol-Oksuz, Y. Afanasyeva, A. Heguy, M. Thompson, K. Ray, A. Giro-Perafita, I. Sánchez, X. Wu, D. Tripathy, A. Zeleniuch-Jacquotte, A. Tsirigos, and F.J. Esteva, Prognostic role of elevated mir-24-3p in breast cancer and its association with the metastatic process. Oncotarget, 2018. 9(16): p. 12868-12878.

46. Sun, X., D. Xiao, T. Xu, and Y. Yuan, miRNA-24-3p promotes cell proliferation and regulates chemosensitivity in head and neck squamous cell carcinoma by targeting CHD5. Future Oncol, 2016. 12(23): p. 2701-2712.

47. Meng, F.L., W. Wang, and W.D. Jia, Diagnostic and prognostic significance of serum miR24-3p in HBV-related hepatocellular carcinoma. Med. Oncol., 2014. 31(9): p. 177.

48. Yang, Y., B. Jia, X. Zhao, Y. Wang, and W. Ye, miR-93-5p may be an important oncogene in prostate cancer by bioinformatics analysis. J. Cell. Biochem., 2019. 120(6): p. 1046310483.

49. Li, L., J. Zhao, S. Huang, Y. Wang, L. Zhu, Y. Cao, J. Xiong, and J. Deng, MiR-93-5p 
promotes gastric cancer-cell progression via inactivation of the Hippo signaling pathway. Gene, 2018. 641: p. 240-247.

50. Ma, D.H., B.S. Li, J.J. Liu, Y.F. Xiao, X. Yong, S.M. Wang, Y.Y. Wu, H.B. Zhu, D.X. Wang, and S.M. Yang, miR-93-5p/IFNAR1 axis promotes gastric cancer metastasis through activating the STAT3 signaling pathway. Cancer Lett., 2017. 408: p. 23-32.

51. Xiang, Y., X.H. Liao, C.X. Yu, A. Yao, H. Qin, J.P. Li, P. Hu, H. Li, W. Guo, C.J. Gu, and T.C. Zhang, MiR-93-5p inhibits the EMT of breast cancer cells via targeting MKL-1 and STAT3. Exp. Cell Res., 2017. 357(1): p. 135-144.

52. Li, J., Z.P. Chu, H. Han, Y. Zhang, F. Tian, J.Q. Zhang, and X.H. Huang, Suppression of miR-93-5p inhibits high-risk HPV-positive cervical cancer progression via targeting of BTG3. Hum. Cell, 2019. 32(2): p. 160-171.

53. Wang, X., Z. Liao, Z. Bai, Y. He, J. Duan, and L. Wei, MiR-93-5p Promotes Cell Proliferation through Down-Regulating PPARGC1A in Hepatocellular Carcinoma Cells by Bioinformatics Analysis and Experimental Verification. Genes (Basel), 2018. 9(1).

54. Simpson, R.J., J.W. Lim, R.L. Moritz, and S. Mathivanan, Exosomes: proteomic insights and diagnostic potential. Expert Rev Proteomics, 2009. 6(3): p. 267-83.

55. Jeppesen, D.K., A.M. Fenix, J.L. Franklin, J.N. Higginbotham, Q. Zhang, L.J. Zimmerman, D.C. Liebler, J. Ping, Q. Liu, R. Evans, W.H. Fissell, J.G. Patton, L.H. Rome, D.T. Burnette, and R.J. Coffey, Reassessment of Exosome Composition. Cell, 2019. 177(2). 
Figure 1

Overview of the experiment design.

PC: prostate cancer; NC: normal control.

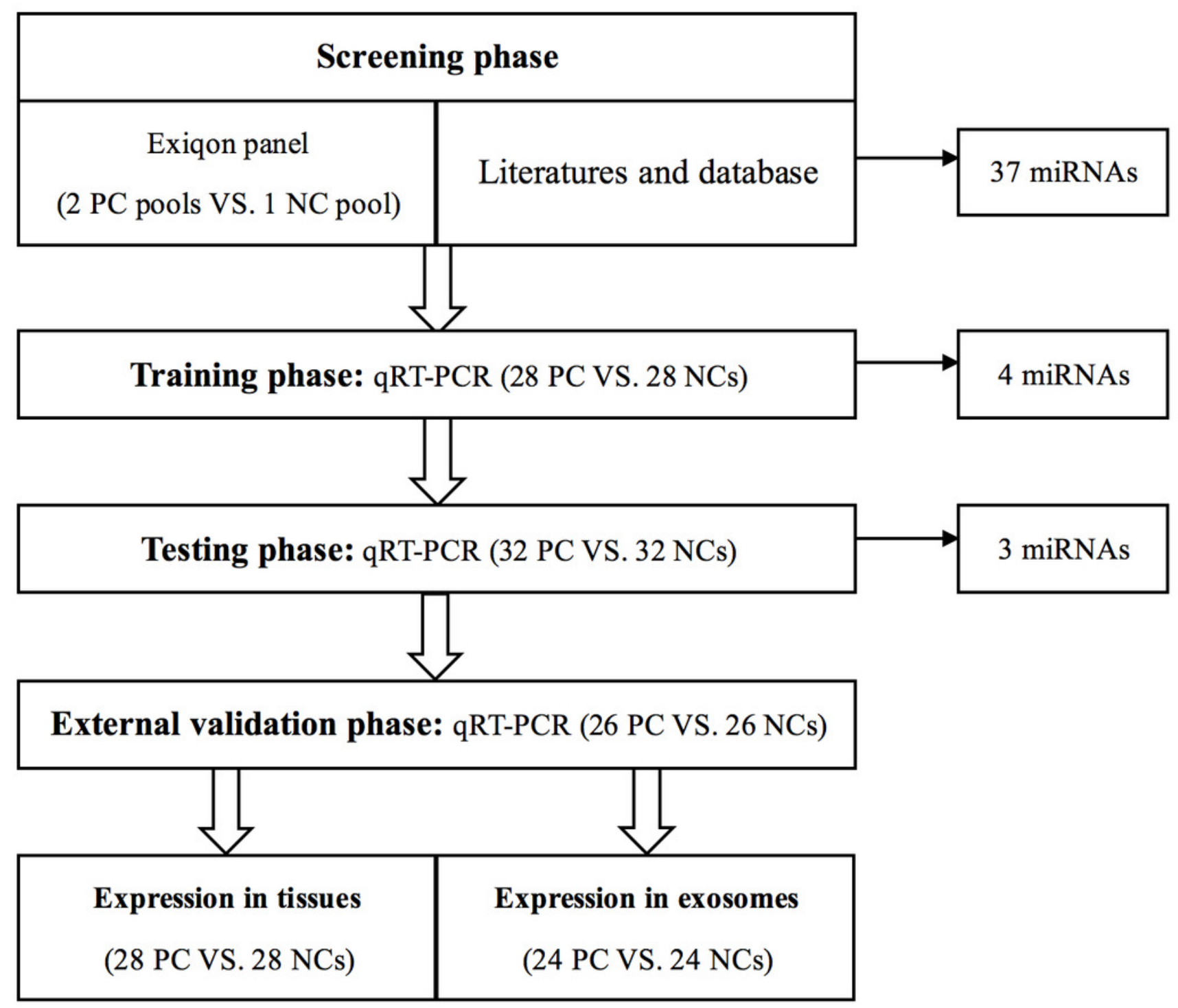


Figure 2

Expression on levels of three miRNAs in serum of 60 PC patients and 60 controls (in the training and testing stages).

(A) miR-146a-5p. (B) miR-24-3p. (C) miR-93-5p.

PC: prostate cancer; T: tumor ; N: normal control. Horizontal line: mean with 95\% Cl.
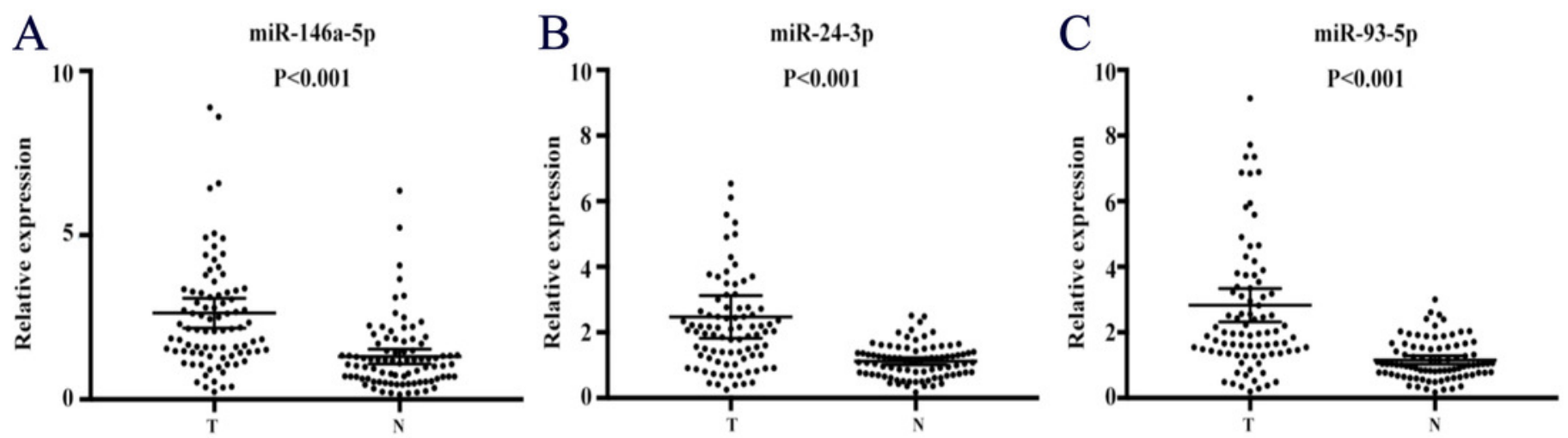


\section{Figure 3}

Receiver-operating characteristic (ROC) curve analyses of the three-miRNA signature to discriminate PC patients from normal controls.

(A) the combined three cohorts of training, testing, and validation stages (86 PC vs. 86 NCs).

(B) the combination of the former two cohorts (the training and testing stage; 60 PC vs. $60 \mathrm{NCs}$ ).

(C) external validation stage (26 PC vs. 26 NCs).

PC: prostate cancer; NC: normal control; AUC: areas under the curve.
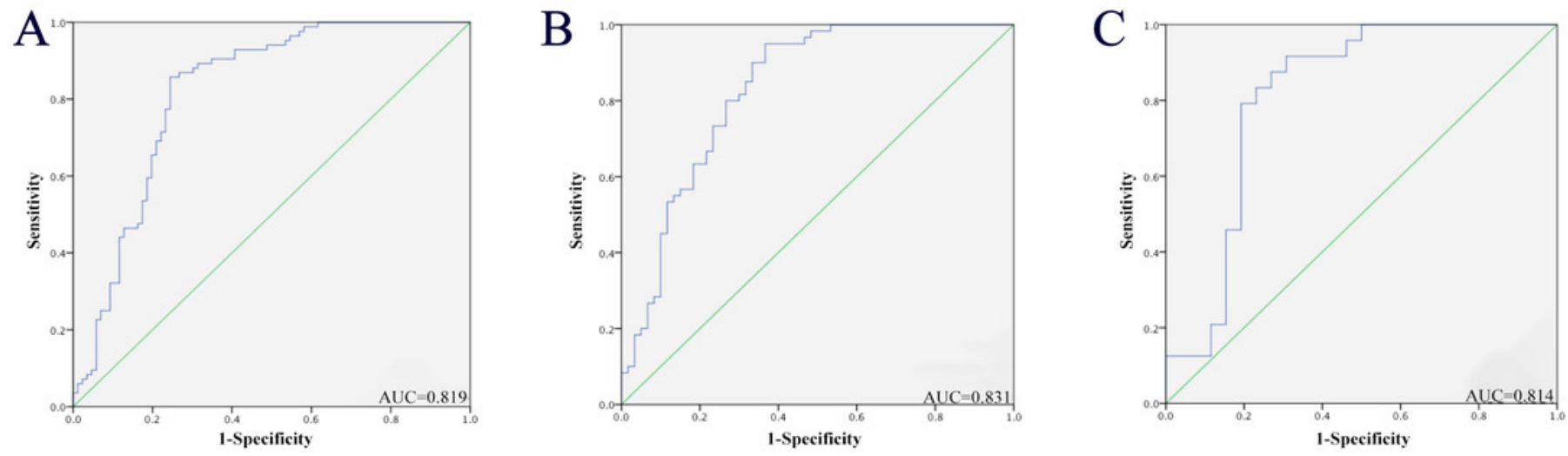
Figure 4

Prostate cancer diagnostic capability of the three-miRNA panel verified in the four public datasets.

(A) TCGA: AUC $=0.979,95 \% \mathrm{Cl}: 0.967-0.991, \mathrm{P}<0.001$, Sensitivity $=94.1 \%$, Specificity $=94.0 \%$.

(B) GSE113740: AUC $=0.806,95 \%$ Cl:0.733-0.880, P<0.001, Sensitivity $=84.0 \%$, Specificity $=68.7 \%$.

(C) GSE113486: AUC $=0.943,95 \% \mathrm{Cl}: 0.901-0.986, \mathrm{P}<0.001$, Sensitivity $=42.5 \%$, Specificity $=100.0 \%$.

(D) GSE112264: AUC $=0.950,95 \% \mathrm{Cl}: 0.925-0.976, \mathrm{P}<0.001$, Sensitivity $=86.2 \%$, Specificity $=92.7 \%$.
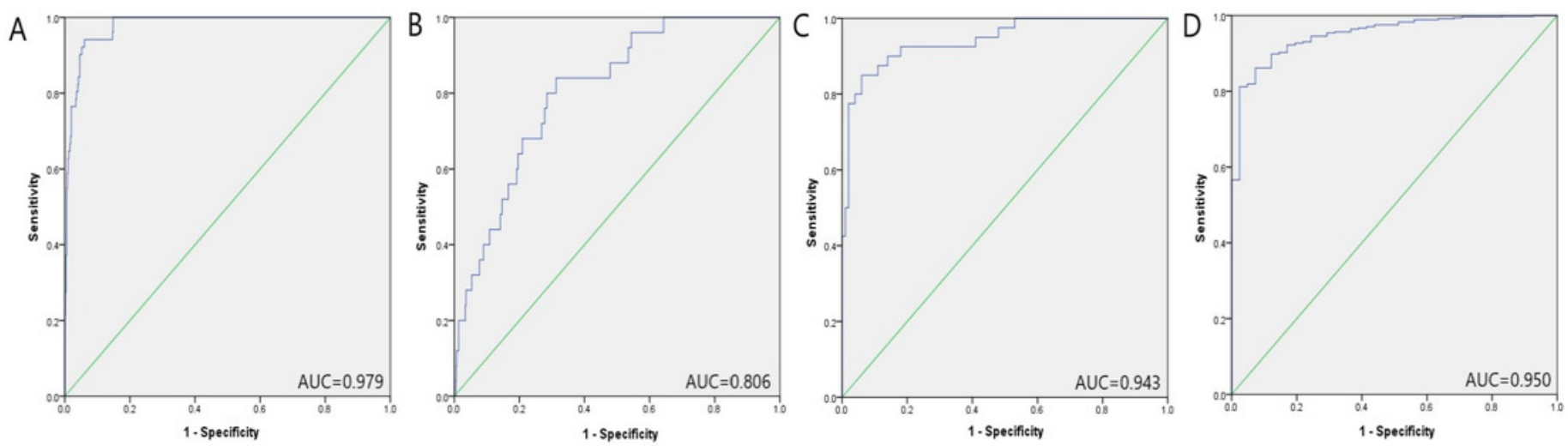
Figure 5

Expression of the three miRNAs (data from TCGA).

(A) miR-146a-5p. (B) miR-24-3p. (C) miR-93-5p.

T: tumor; N: normal control; TCGA: the cancer genome atlas.

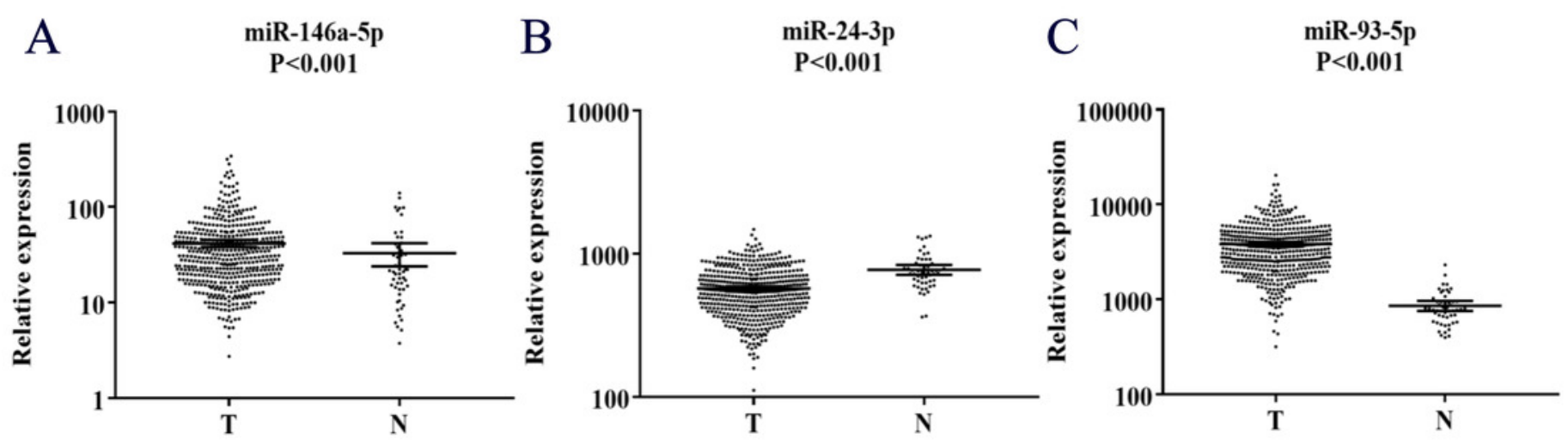


Figure 6

Expression of the three miRNAs in the serum exosome samples of 24 PC patients and 24 NCs.

(A) miR-146a-5p. (B) miR-24-3p. (C) miR-93-5p.

T: tumor; N: normal control.
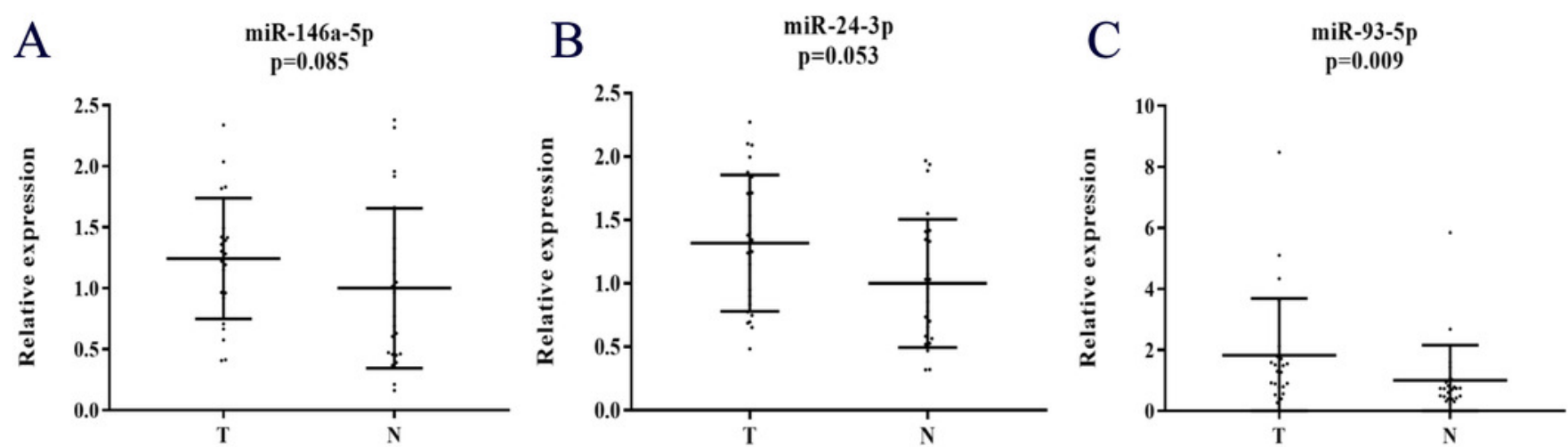


\section{Table $\mathbf{1}$ (on next page)}

Clinical characteristics of 86 PC patients and 86 healthy controls enrolled in the study. fPSA*: free prostate-specific antigen; tPSA*: total prostate-specific antigen. 
1

\begin{tabular}{|c|c|c|c|c|c|c|}
\hline \multirow[t]{2}{*}{ Variables } & \multicolumn{2}{|c|}{$\begin{array}{l}\text { Training stage } \\
\quad(n=56)\end{array}$} & \multicolumn{2}{|c|}{$\begin{array}{l}\text { Testing stage } \\
(n=64)\end{array}$} & \multicolumn{2}{|c|}{$\begin{array}{c}\text { External validation } \\
\text { stage } \\
(n=52) \\
\end{array}$} \\
\hline & Cases (\%) & $\begin{array}{l}\text { Controls } \\
(\%)\end{array}$ & Cases (\%) & $\begin{array}{l}\text { Controls } \\
(\%)\end{array}$ & Cases (\%) & $\begin{array}{l}\text { Controls } \\
(\%)\end{array}$ \\
\hline Number & 28 & 28 & 32 & 32 & 26 & 26 \\
\hline \multicolumn{7}{|l|}{ Age } \\
\hline$<70$ & $14(50.0)$ & $12(42.9)$ & $15(46.8)$ & $16(50.0)$ & $11(42.3)$ & $12(46.2)$ \\
\hline$\geq 70$ & $14(50.0)$ & $16(57.1)$ & $17(53.1)$ & $16(50.0)$ & $15(57.7)$ & $14(53.8)$ \\
\hline \multicolumn{7}{|l|}{$\begin{array}{l}\text { Gleason } \\
\text { grade }\end{array}$} \\
\hline$\leq 7$ & $12(42.9)$ & & $18(56.3)$ & & $13(50)$ & \\
\hline$\geq 8$ & $16(57.1)$ & & $14(43.7)$ & & $13(50)$ & \\
\hline \multicolumn{7}{|l|}{ TNM stage } \\
\hline I & $5(17.8)$ & & $7(21.9)$ & & $6(23)$ & \\
\hline II & $6(21.4)$ & & $9(28.1)$ & & $5(19.2)$ & \\
\hline III & $12(43)$ & & $9(28.1)$ & & $8(30.8)$ & \\
\hline $\mathrm{IV}$ & $5(17.8)$ & & 7 (21.9) & & $7(27)$ & \\
\hline \multicolumn{7}{|l|}{$\begin{array}{l}\text { fPSA* }^{*} \\
\quad(\mathrm{ng} / \mathrm{ml})\end{array}$} \\
\hline$<0.5$ & $8(28.6)$ & & $10(31.2)$ & & $8(30.8)$ & \\
\hline$\geq 0.5, \quad<5$ & $6(21.4)$ & & $11(34.4)$ & & $11(42.3)$ & \\
\hline$\geq 5$ & $14(50)$ & & $11(34.4)$ & & $7(26.9)$ & \\
\hline \multicolumn{7}{|l|}{ tPSA* } \\
\hline$<4$ & $4(14.3)$ & & $5(15.6)$ & & $2(7.6)$ & \\
\hline$\geq 4, \quad<40$ & $7(25)$ & & $14(43.8)$ & & $12(46.2)$ & \\
\hline$\geq 40$ & $17(60.7)$ & & $13(40.6)$ & & $12(46.2)$ & \\
\hline \multicolumn{7}{|c|}{ fPSA/tPSA } \\
\hline$<0.25$ & $25(89.3)$ & & $29(90.6)$ & & $25(96.2)$ & \\
\hline$\geq 0.25$ & $3(10.7)$ & & $3(9.4)$ & & $1(3.8)$ & \\
\hline
\end{tabular}

2 


\section{Table 2 (on next page)}

MiRNAs that are differently expressed in the two PC pools in the screening phase.

Each ten samples are mixed as one pool sample. 


\begin{tabular}{|c|c|c|c|}
\hline \multirow{2}{*}{ MiRNA } & \multicolumn{2}{|c|}{ Fold change } & \multirow{2}{*}{ Mean fold } \\
\hline & Pool 1 & Pool 2 & \\
\hline hsa-let-7g-5p & 1.562103 & 1.516144 & 1.5391235 \\
\hline hsa-miR-1-3p & 1.592673 & 3.164749 & 2.378711 \\
\hline hsa-miR-10b-5p & 1.753225 & 11.63021 & 6.6917175 \\
\hline hsa-miR-126-3p & 1.888629 & 2.330068 & 2.1093485 \\
\hline hsa-miR-144-5p & 2.786662 & 3.532729 & 3.1596955 \\
\hline hsa-miR-152-3p & 1.808996 & 1.756634 & 1.782815 \\
\hline hsa-miR-155-5p & 4.610215 & 3.432634 & 4.0214245 \\
\hline hsa-miR-15b-3p & 1.727401 & 1.952506 & 1.8399535 \\
\hline hsa-miR-195-5p & 5.29328 & 3.112925 & 4.2031025 \\
\hline hsa-miR-28-5p & 2.37564 & 2.142608 & 2.259124 \\
\hline hsa-miR-296-5p & 1.838551 & 2.485472 & 2.1620115 \\
\hline hsa-miR-29a-5p & 4.160826 & 2.80201 & 3.481418 \\
\hline hsa-miR-30a-5p & 1.511533 & 1.824362 & 1.6679475 \\
\hline hsa-miR-30e-3p & 1.563817 & 2.235089 & 1.899453 \\
\hline hsa-miR-335-5p & 2.034361 & 2.060766 & 2.0475635 \\
\hline hsa-miR-551b-3p & 1.818341 & 8.612135 & 5.215238 \\
\hline hsa-let-7a-5p & 1.53924 & 1.680264 & 1.609752 \\
\hline hsa-miR-103a-3p & -3.57075 & -4.40626 & -3.988505 \\
\hline has-miR-106a-5p & 2.061031 & 2.266201 & 2.163616 \\
\hline hsa-miR-141-3p & -44.0872 & -36.1883 & -40.13775 \\
\hline hsa-miR-16-5p & 1.60983 & 1.780559 & 1.6951945 \\
\hline hsa-miR-21-5p & 2.174586 & 1.516818 & 1.845702 \\
\hline hsa-miR-210-3p & 1.577939 & 2.310396 & 1.9441675 \\
\hline hsa-miR-223-3p & 1.674394 & -11.1606 & -4.743103 \\
\hline hsa-miR-24-3p & 1.585616 & 1.875112 & 1.730364 \\
\hline hsa-miR-26b-5p & 1.554088 & -2.66348 & -0.554696 \\
\hline hsa-miR-30c-5pc & -2.05815 & -2.29057 & -2.17436 \\
\hline hsa-miR-375-3p & -1.78726 & -1.81663 & -1.801945 \\
\hline hsa-miR-93-5p & 1.510669 & 1.892198 & 1.7014335 \\
\hline hsa-miR-146a-5p & 1.53914 & 1.651484 & 1.595312 \\
\hline hsa-miR-200a-3p & 6.006406 & 4.104479 & 5.0554425 \\
\hline has-miR-200c-3p & -2.97765 & -1.70005 & -2.33885 \\
\hline has-miR-574-3p & -4.33151 & -6.01175 & -5.17163 \\
\hline hsa-miR-107 & -5.16725 & -6.01989 & -5.59357 \\
\hline hsa-miR-133b & -1.82926 & -1.64685 & -1.738055 \\
\hline hsa-miR-143-3p & -2.76443 & -3.21305 & -2.98874 \\
\hline hsa-miR-221-3p & -2.33089 & -1.75637 & -2.04363 \\
\hline
\end{tabular}

1 


\section{Table 3(on next page)}

Expression levels of the 3 serum mi-RNAs in the training and testing phases

$\mathrm{FC} *$ : fold change. (presented as mean $\pm \mathrm{SD} ; \Delta \mathrm{CT}$ : relative to miR-103a-3p).

The combined column stands for a combination of the two cohorts (the training and testing stages). 


\begin{tabular}{|c|c|c|c|c|c|c|c|c|c|c|}
\hline \multirow{2}{*}{ miRNA } & \multicolumn{4}{|c|}{ Training phase } & \multicolumn{4}{|c|}{ Testing phase } & \multicolumn{2}{|c|}{ Combined } \\
\hline & PC & NC & FC & $P$ value & PC & $\mathbf{N C}$ & FC & $P$ value & $\mathbf{F C} *$ & P value \\
\hline miR-146a-5p & $-3.73 \pm 1.26$ & $\begin{array}{c}-2.99 \pm \\
1.18\end{array}$ & 1.68 & 0.031 & $-3.15 \pm 1$ & $-1.81 \pm 1.15$ & 2.53 & $<0.001$ & 2.24 & $<0.001$ \\
\hline miR-24-3p & $-2.73 \pm 1.11$ & $-2.01 \pm 0.86$ & 1.64 & 0.014 & $-3.40 \pm 0.98$ & $-2.50 \pm 0.6$ & 1.88 & $<0.001$ & 1.81 & $<0.001$ \\
\hline miR-93-5p & $-1.93 \pm 1.36$ & $\begin{array}{c}-0.86 \pm \\
0.76\end{array}$ & 2.1 & 0.002 & $-1.92 \pm 1.11$ & $-0.74 \pm 1.02$ & 2.27 & $<0.001$ & 2.22 & $<0.001$ \\
\hline miR-143-3p & $0.44 \pm 1.4$ & $1.05 \pm 1.37$ & 1.53 & 0.016 & $0.67 \pm 0.96$ & $1.24 \pm 0.89$ & 1.48 & 0.028 & & $\square$ \\
\hline
\end{tabular}

1 